\title{
Past, Present, and Future of Electron Microscopy Chemical Processing Workflows
}

\author{
Steven Goodman, Mark Nelson and Thomas Strader
}

Microscopy Innovations, LLC, Marshfield, Wisconsin, United States

Electron microscopy of biological specimens began with the development of room temperature chemical preparation methods for TEM, receiving the 1974 Nobel Prize for Physiology or Medicine [1]. The workflow to prepare specimens required invention of ultramicrotomes and knifes for ultrathin sectioning, other apparatus, and especially preparative chemistries to stabilize specimens for microtomy, vacuum, and for electron-beam irradiation, and to provide image contrast. These chemical workflows are routine today, including aldehyde fixation, $\mathrm{OsO}_{4}$ post fixation, graded solvent dehydration, serial infiltration in epoxy resins, and metal stains.

Since the 1974 Nobel Prize, there have been remarkable workflow improvements in TEM instruments. These now include automated image acquisition and analysis, and the advent of 3D Electron Microscopies (3DEM) including serial block face, array tomography, and FIB SEM. There have also been major improvements in microtomy and other tools that reduce the time and effort for specimen preparation and improve upon the workflows developed in the 1970s.

Despite instrumentation workflow improvements, there has been much less change in the lab processes to deliver the specimen preparation chemicals. Most specimens are still processed as they were decades ago: by immersion in vials with manual reagent exchanges every few minutes or hours. Typical protocol times are 1-2 days for TEM, plus an additional day for immunogold labeling (IGL), while 3DEM requires 4-5 days (Fig. 1). Hands-on effort is also significant, and is required sporadically, making it problematic to perform other tasks. Microwave processing substantially decreases total preparation times, but requires nearly continuous hands-on effort with manual reagent exchanges every few minutes. Tissue autoprocessors can prepare large numbers of specimens with less labor. These, however, are not suitable for the more complex and longer IGL or 3DEM protocols. As a result, time-consuming manual effort is required, preventing personnel from performing other work for long periods.

Around the time the 1974 Nobel was awarded for biological EM, a revolution was occurring in the apparatus used for molecular and cell biology. This was the advent of multi-channel pipettors, microwell plates and, somewhat later, fluid handling "robots." In 2010, the mPrep system was introduced to leverage these tools for the EM lab. The mPrep System entraps individual specimens or grids in flow-through capsules that attach to pipettors or to the purpose-built EM preparation "robot", the ASP-1000. The mPrep System enables nearly any protocol, including standard TEM preparation [2], both pre- and postembedding IGL [3], and 3DEM preparation [4]. Automation enables a protocol to be programmed, setup, and "run" without human intervention for hours or days, or to include automated prompts when interventions are required, such as adding a fresh reagent. Further, near continuous reagent flow can be programmed to accelerate diffusion into specimens. This enables renal biopsy TEM specimens to be ready for resin curing in $2 \mathrm{hrs}$, including en bloc Ur staining [2], and to prepare 3DEM specimens as rapidly as one day [4]. Automatic operation and increased processing speed improves workflow by freeing personnel (Fig. 1), and also by reducing the potential for human error.

Where can we look to future workflow enhancements for chemical preparation? EM labs spend considerable time preparing reagents from raw chemicals. Workflows would be improved with easier-touse reagent "kits and new formulations. These would save time, improve reproducibility, and could lead 
to some standard protocols. Automation can be used to further optimize protocols for reliability, throughput, and to reduce personnel time.

Chemical processing has been the most robust method to prepare biological specimens for half a century. We can expect it will continue to advance biology and biomedicine in concert with advances in TEM, and the increased implementation of 3DEM. As workflow efficiencies for TEM and 3DEM continues to improve, including automated imaging and analysis, we can anticipate the need to prepare ever more specimens. This will require further preparation workflow improvements, and the need for consistent sample management and documentation.

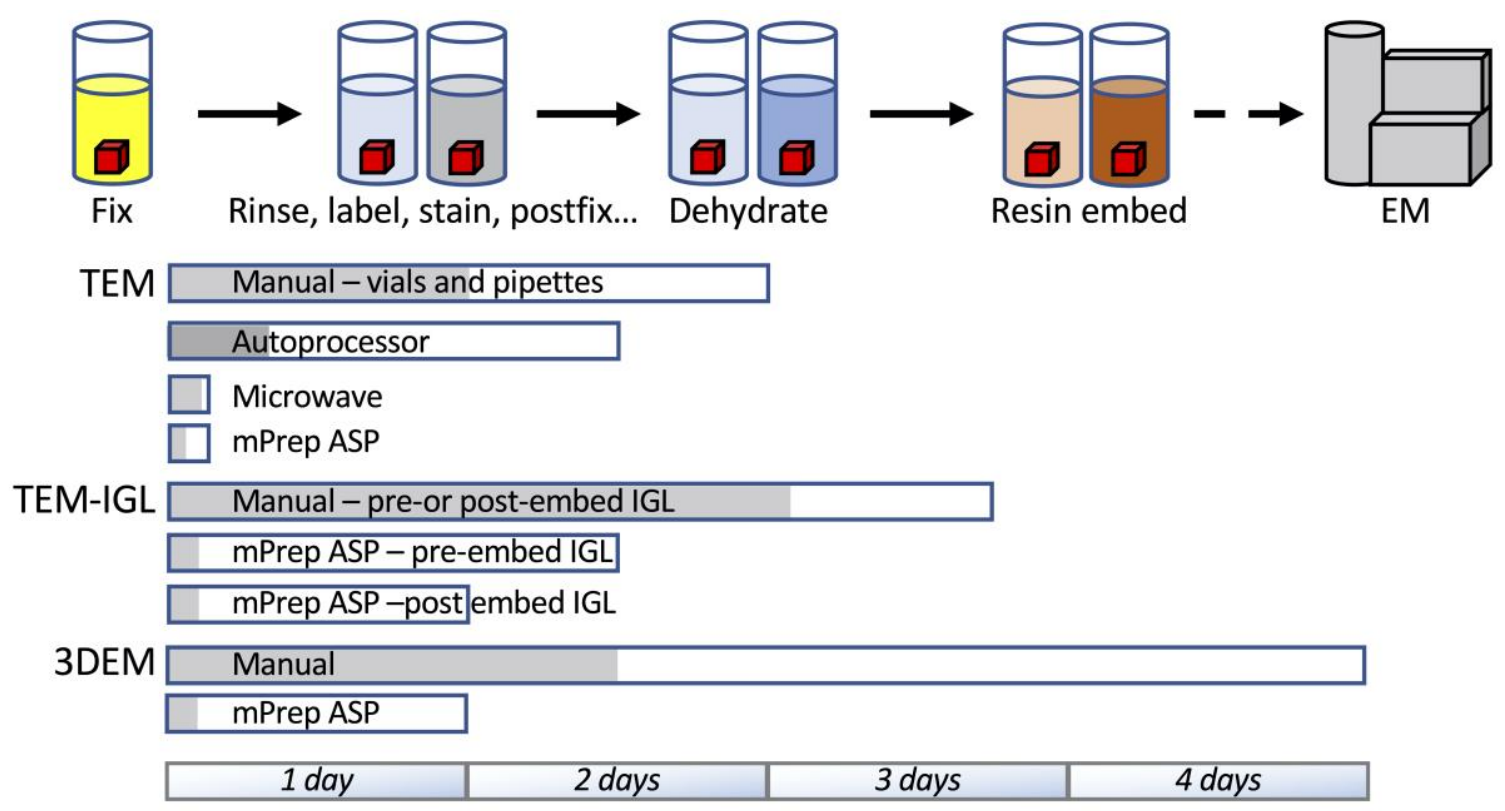

Figure 1. Reagent processing workflow efficiency for TEM, immunogold TEM, and 3DEM with different methods. Bar length shows typical protocol times for specimens after initial aldehyde fixation until ready for final resin cure. Gray bars indicate approximate cumulative hands-on effort. In practice, hands-on effort occurs sporadically over the entire protocol time.

\section{References}

1. Nobel Prize: "Discoveries concerning the structural and functional organization of the cell": https://www.nobelprize.org/prizes/medicine/1974/summary/

2. TE Strader, BK August, Microsc. Microanal 25 (2019) p. 1184.

3. P Marques et al., Microsc. Microanal 24 (2018) p. 1300.

4. SL Goodman, et al. Soc. Neuroscience, (2019) 429.02/CC50. 